\title{
A Gadget Recommendation System using Data Science
}

\author{
A Pushpalatha*, Harish Sanmugam J, Jeya Pradeepa, Madhu Bala S \\ Department of Computer Science and Engineering, Sri Krishna College of Engineering and \\ Technology, Coimbatore, India \\ E-mail: * pushpalathaa@skcet.ac.in
}

\begin{abstract}
Electronic gadgets have become vital in basic need of individual. The advances in technology, makes it necessary to satisfy different functional needs of users. Therefore, it is important to suggest gadgets for users according to personal preference and various constraints of electronic gadgets. We propose a gadget recommendation system by integrating various requirements from users.
\end{abstract}

Keywords- Gadget recommendation, classification, model rendering, k-means algorithm, content-based filtering.

\section{$1 \quad$ Introduction}

Today, gadgets have become basic need of an individual, as a communication and entertainment device across the globe. The tech world has become more competitive than ever with a lot of manufactures trying to produce the best for their customers. The endless increase in the options space left the customers in a dilemma of choosing the right gadget for them. The major factors that influence customers in selecting an electronic gadget to use include: brand, RAM, battery life, price, year of release, innovative features, personal recommendation and so on. Though most electronic gadgets have all the basic features, manufactures still try to bring uniqueness into their products by adding some more new features to the existing features to make them stand out of the competition. The manufactures accepted the challenge and competition of making a perfect gadget for their targeted customers by implementing innovative ideas and features to enhance the user experience. The growing number of brands and models has created a fierce market competition. Therefore, the users are left with a lot of options to pick from.

This work started with the introduction of user preference base approach, creating of custom data set, application of proposed approach on the data sets and the justification of the importance of the proposed methodology through comparative discussion.

\section{Literature Survey}

There are E-commerce websites available for each gadget, but it doesn't recommend the best according to the user's interest. $91 \%$ of shoppers are bound to shop with brands who perceive, recollect and give pertinent offers and suggestions, which is not available in the existing system. This is where our product stands-out of the market crowd.

\section{A. Problems in Existing System}

(i) Solely based on mobile or laptop and not wide variety of gadgets

(ii) Does not suggest based on user preferences.

(iii) Most of them uses collaborative filtering which mimics user to user recommendation.

\section{Related Work}

We propose an AI based recommendation system specially designed for electronic gadgets. It has a simple UI with many functions. It is a user-friendly app with profile free interaction. It provides a superior customer experience that can deliver the level of personalization which will impress consumers with individualized options. We collected a wide range of data and cleaned it using data pre-processing. Then we specially tuned K-means algorithm to match the user input using wide range of collected data. 
Journal of Information Technology and Digital World (2020)

Vol.02/ No. 04

Pages: 213-216

https://www.irojournals.com/itdw/

DOI: https://doi.org/10.36548/jitdw.2020.4.004

TECH SENSEI

GADGET RECOMMENDATION SYSTEM

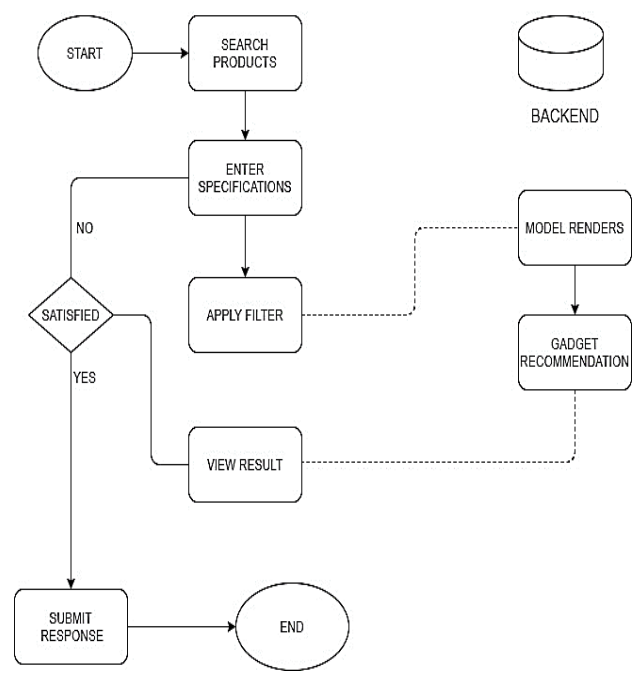

A. Description

Fig. 1. Architecture Diagram

Gadget recommendation system is a software tool designed to give recommendations to things or substance a particular user would like to purchase or engage with. Firstly, the user logs in. Secondly, the user searches for gadgets according to his preference by entering certain specifications in the filter option. If the user is satisfied with the result/ recommended gadget he submits a response and logs out. Else he repeats the second step.

Step 1: The user searches for the required product.

Step 2: The user can enter specifications to get more accurate result.

Step 3: After applying the filter, the trained model fetches the result.

Step 4: The fetched result is displayed to the user.

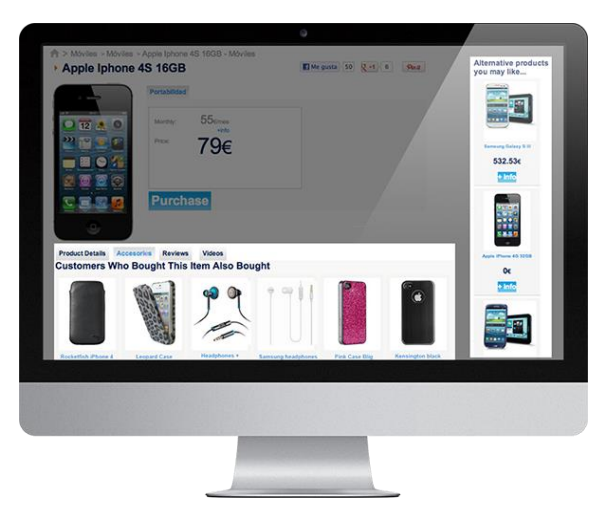

Fig. 2. A Gadget Recommendation 
Vol.02/ No. 04

Pages: $213-216$

https://www.irojournals.com/itdw/

DOI: https://doi.org/10.36548/jitdw.2020.4.004

$\begin{array}{ll}\text { B. } & \text { Methodology } \\ \text { 1. } & \text { Flutter } 1.17\end{array}$

Flutter is an Google's mobile UI framework for crafting high performing modern apps. The user interface is built with widgets using a technique called composition. It is performant tool when it comes to adding new features or fixing bugs, making it a highly appealing framework.

2. Python Libraries

2. A. SCIKIT LEARN

It is a free software machine learning library for the Python programming language. It provides various clustering and classification algorithm. It is framed to collaborate and function with python libraries.

\section{B. NUMPY}

NumPy is a python library which is used for scientific operations. The ndarray is multidimensional array which stores values of same data type. It helps to integrate with the databases.

\section{C. PANDAS}

Pandas is a high-performing, easy to use open source tool developed for data manipulation by Wes McKinney. It is built on the Numpy package. Data Frames, which is its key data structure allows you to gather data and manipulate tabular data in rows of observations and columns of variables.

\section{D. JUPYTER NOTEBOOK}

The Jupyter Notebook is a powerful, flexible tool, which provides web-based application that allows you to develop, document, execute code with visualizations and narrative texts.

\section{Results And Discussions}

Wide range of content and data were collected and custom data sets were made. K-means algorithm was specially tuned to match the user input using the wide range of extracted data which was pre-processed. Then models were trained and validated to develop a product for deployment. UX is built to create products which involve acquiring and integrating the product, which features brand, usability design and function. In unit testing we test the functions to ascertain if they are fit to use and asserts a certain behavior or state. This testing ensures that the source code written by us meets the requirement and behaves in an expected manner. In market fit stage, the target customer is identified and served with the right product. Then, the next step is to scale by finding more customers within the target market. Depending on the position in the market, it is integrated within the ecosystem.

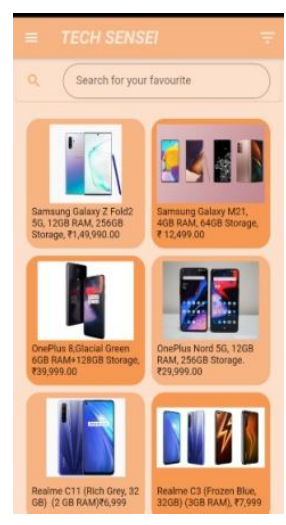

Fig. 3. Search result for phone recommendation 
Vol.02/ No. 04

Pages: $213-216$

https://www.irojournals.com/itdw/

DOI: https://doi.org/10.36548/jitdw.2020.4.004

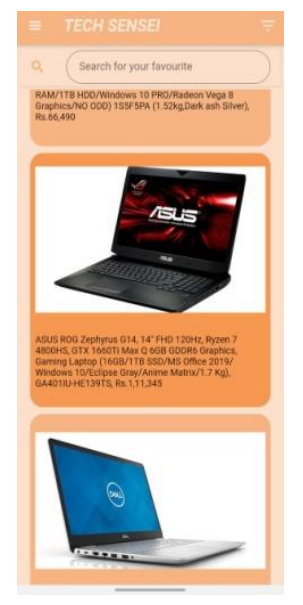

\section{Conclusion}

Fig. 4. Search Result for laptop recommendation

Machine learning techniques are used to collect and store data about every gadget and users to create a connection between those users and the gadgets. In this work, for gadget selection a user preference methodology was implemented. Thus, the important issue of how to discern what device users need to enrich and satisfy their personal need from in depth personalization service has been solved in the field of personalized recommendation systems. The results show that a gadget recommendation system can effectively improve the quality of services.

\section{Future Scope}

Electronic gadgets have become vital in basic need of individual. The advances in technology, makes it necessary to satisfy different functional needs of end-users. Therefore, it is significant to suggest gadgets for the customers based on their personal preference. Recently with rapid development of technology, smart devices and communication networks spring up to cover all the aspect of customer's activity. These data can also be trained and modelled for future use to cope with the upcoming technological innovations.

\section{References}

1. Y.Subba Reddy and Govindarajulu, A mobile phone recommendation system with user centric voting approach, 2018

2. Pegah malekpour alamdari; nima jafari navimipour), a systematic study on the recommender systems in the e-commerce, 2020

3. Saman Forouzandeh, Recommender system for Users of Internet of Things (IOT),2020

4. Shahab Saquib Sohail ,Classifications of Recommender Systems: A review, 2017

5. Pradeep Kumar Singh, Recommender Systems: An Overview, Research Trends and Future Directions,2020

6. R. Venkatesan1, A. Sabari," Issues in various Recommenders System in E-Commerce-A Survey,2020 